\title{
Greenhouse Environment Monitoring System Design and Implementation
}

\author{
Lifen Wang
}

Jilin College of Agricultural Science and Technology Information Engineering, Jilin 132101, China

wlf800@126.com

Keywords: Microcontroller; Tempreture \& humidity; Sensor; System

\begin{abstract}
The supervising system of flower house's enviroment, which is put into function by the popul. Ar series of AT89S52 single machine tablet, mainly measures and controls the major tempreture and Humidity of enviroment, which will be revealed by showing system. The paper gives an introduction of System's software, hardware design and the process of performing. The system is easily manured with Great utility.at the same time, it worths being popularized and used.
\end{abstract}

\section{Introduction}

Flower Greenhouse in china started late but developed rapidly to the forefront around the world. Automatic greenhouse controlling system, suiting our country's condition, is imminent. The design is based on temperature and humidity measurement and control systems of single-chip [1], modular and hierarchical ways. New Intelligent temperature and humidity sensor-DHT11, which does research on temperature and humidity, and controls them, will collect and change temperature and humidity signal into digital signal, then makes analysis and process of data by single-chip AT89S52 to show LCD Monitor with partial character LCD1602.The system [2] solves many problems in manually controlled flower greenhouse, such as big errors of temperature and humidity, cost of time and labor and low efficiency. It has precise control of the growth of flowers, increases production, and brings economic and social benefits.

\section{Design of the System Plans}

Environmental Facilities output program according to Environment models needed for crop growth is the key skill to control greenhouse environment. In avoidance of complete controlling plans, this type chooses the most important environmental factors, such as temperature, humidity, light and the density of co2 in greenhouse, as the basic monitoring and controlling project, and designs the overall system plans like chart 1 , which is based on sunny greenhouse' own character.

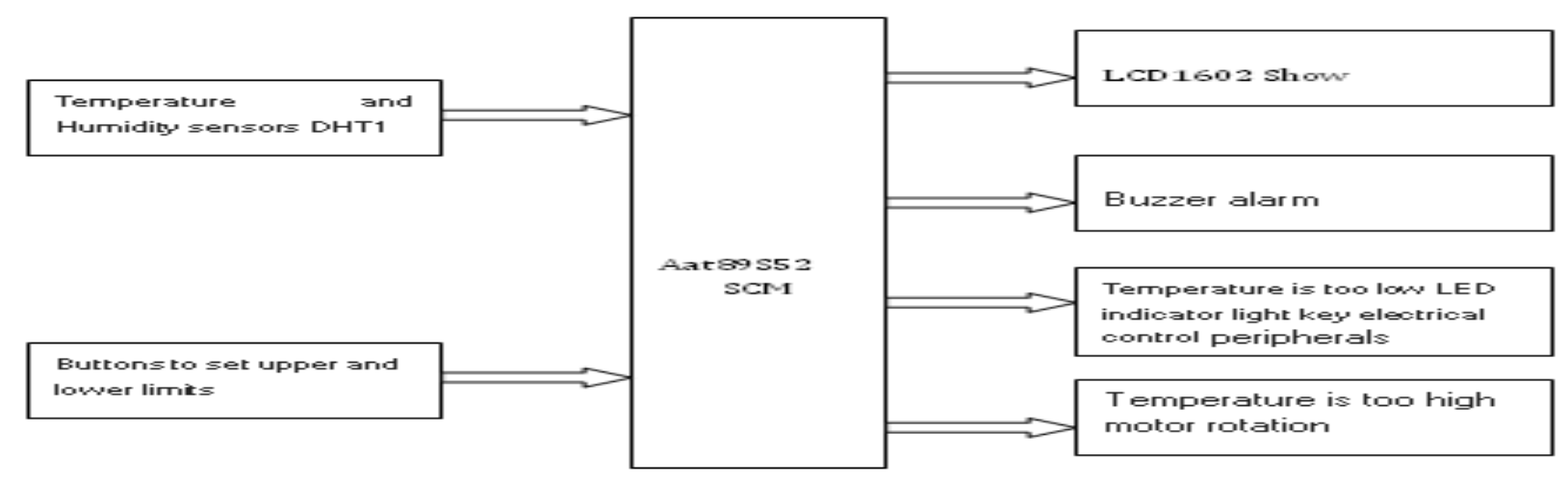

Figure 1. Overall system design 
The design of the core components of the system AT89S52, signal acquisition and processing part constituted by the DHT11, after treatment by LCD1602 display temperature and humidity after entering the microcontroller[3]. In the software design of the temperature and humidity measurements have set the upper and lower values when measuring value exceeds the limit by limit alarm processing circuit to process it show different diode lights, buzzer sounding generated. Hardware includes a switch, the reset switch [4]. When turned on, all device initialization, temperature and humidity sensors DHT11 begin measurement and calculation of temperature and humidity, the final result will be displayed through the LCD display. Has a lower limit than the set temperature and humidity in the measurement results, the module responds by overrun. System circuit shown in Fig. 2:

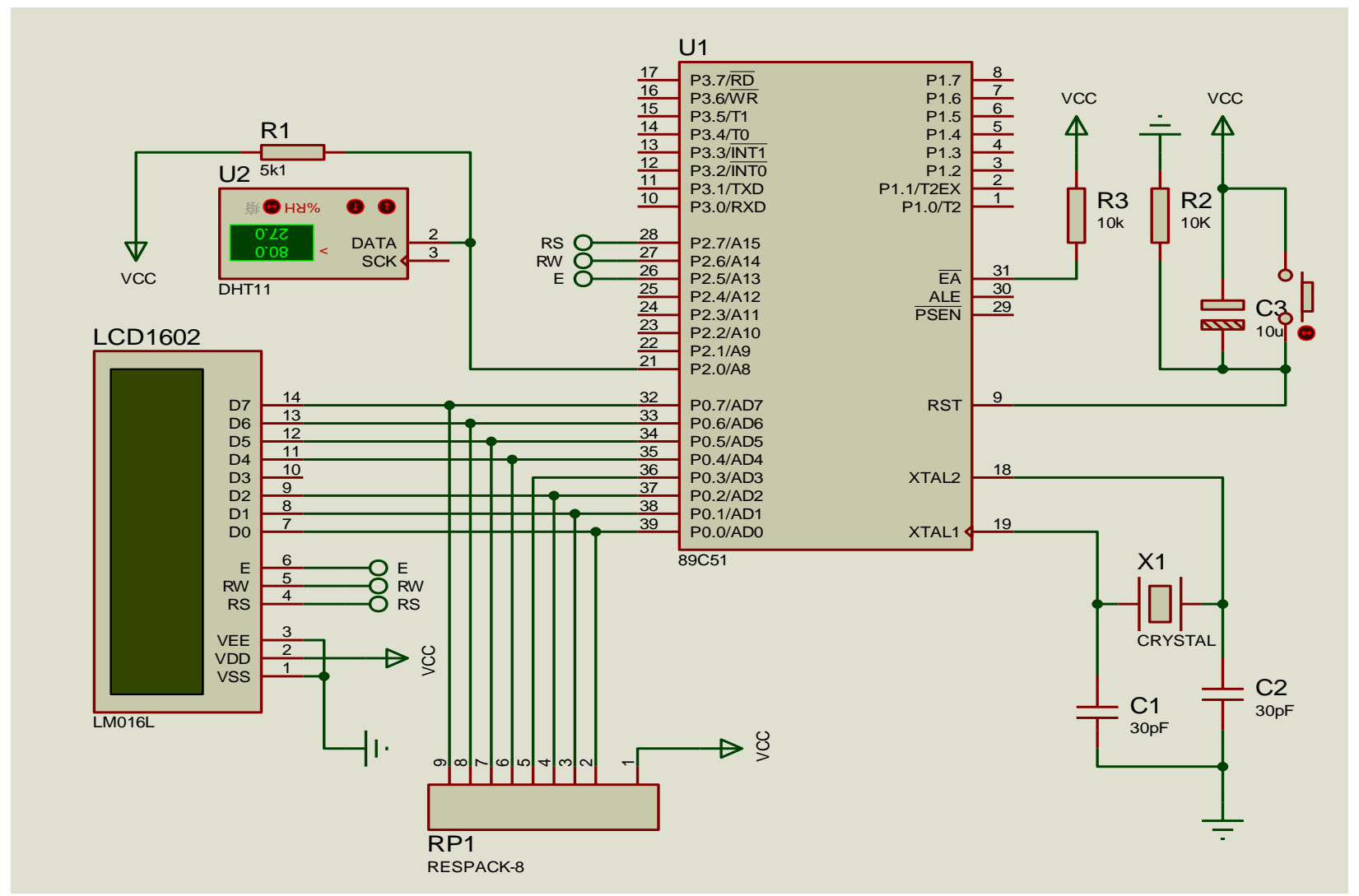

Figure 2. Stem circuit diagram

\section{Hard Wear System Design}

Master Cheapat89s52. Master cheapat89s52, made by High-density nonvolatile memory technology, is a micro controller with low power consumption and high quality. It has following functions: 8k character flash, 256 character, $32 \mathrm{I} / \mathrm{O}$ port lines, watchdog timer and two data pointers, three 16-bit timer / counters, a six-vector two interrupt structure and full-duplex serial port[5].

Display LCD1602 Principle. 1602 takes the standard 16-pin connector with LCD module inside controller that has 11 pieces of controlling orders, and its inner CGROM that stores 160 different graphic dot matrix characters, such as Arabic numerals, capital letters, commonly used symbols, and Japanese kana. Every character has the fixed code, for example, 01000001B is for A, which can be seen by being displayed by modules in the address $41 \mathrm{H}$ dot character graphics [6].

This module is part of the circuit chips by the LCD1602 display real-time temperature and humidity, which MCU P0.0 P0.2 pin LCD1602 4 to 6 pin connected, because there is no AT89S52 MCU P0 
port pull-up resistor, so they indirectly a $5.1 \mathrm{kohm}$ resistor, read and write functions are data. SCM P2.0 P2.8 pin to 14-pin 7 LCD1602 connected for data communications transmission. As shown in Fig. 3.

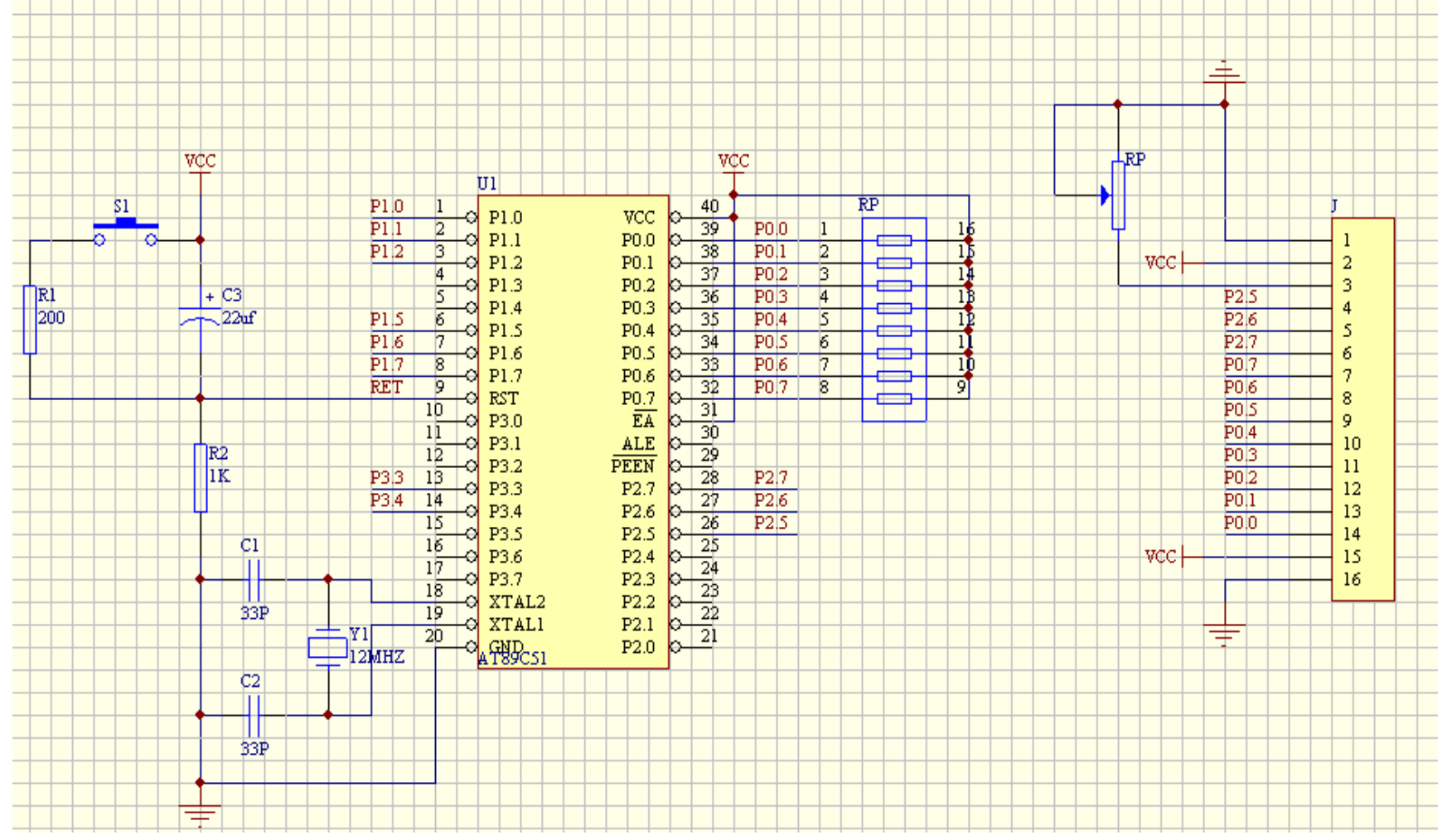

Figure 3. LCD and MCU interface hardware connection diagram

Temperature and Humidity Sensors DHT11. The main function of sensor is to transform model signal collected by sensor into the one that greenhouse controller needs.in Greenhouse environmental parameters, sensor stays where plants need generally by Twisted pair transmitting a signal detected to the greenhouse controller. Concerning with the distance, current in my design has been limited from 0ma to 10ma to reduce the disturb of transmission and ensure the accuracy and reliability of samples. DHT11 digital temperature and humidity sensor is a kind of Temperature and humidity combined sensor with calibrated digital signal output.it is used for special Digital module techniques and temperature and humidity sensor acquisition technology to make sure the extraordinary reliability and long-term stability. Sensor, which connects a high-performance 8-bit micro controller, includes a resistive element and a sense of wet NTC temperature measurement devices. Therefore, the product has excellent quality, fast response, anti-interference ability, high cost, etc. [7] Each DHT11 sensors are calibrated in a precision humidity calibration chamber. Calibration coefficient, saved as a form of program in OTP memory, will be used when internal sensor is detecting signal. Single-wire serial interface of DHT11 sensor makes System Integration easy and quick, and its advantages, such as small volume, low power consumption and over 20-meters signal transmission make itself the best choice for all occasions even the most hash ones. Products have a characteristic of the 4-pin single row pin package, easy connection and Special Package supplied according to customs' needs. When the temperature from DH11T exceeds the prescribed one that alarm sets, buzzer begins to alarm. 


\section{Soft Wear Design}

The program is designed by $\mathrm{C}$ language [8]. After commissioning the software part, the results can be displayed correctly. Specific module consists of a $\mathrm{C}$ (kellen. C) main function files and documents constitute 6 h. [9]

$\mathrm{H}$ files are as follows:

reg51.h; (8051 related parameters initialization)

Absacc.h; Intrins.h; (pseudo eigenfunctions)

Math.h; (math functions)

LCD1604.h; (1604 LCD function)

DHT11.h; (temperature and humidity acquisition calculation function)

set_key.h; (limit alarm function)

The main function analytic

\#include <reg51.h>

\#include <intrins.h>

\#include <key.h>

\#define uchar unsigned char

\#define uint unsigned int

typedef unsigned char unint8;

typedef unsigned char unint16;

sbit $\mathrm{RS}=\mathrm{P} 2^{\wedge} 5$;

sbit $\mathrm{RW}=\mathrm{P} 2^{\wedge} 6$;

sbit $\mathrm{EN}=\mathrm{P} 2^{\wedge} 7$;

sbit $\mathrm{BEEP}=\mathrm{P} 3^{\wedge} 4$;

sbit JDQ=P1^4;

sbit $\mathrm{DJ}=\mathrm{P} 3^{\wedge} 6$;

unsigned char str1[]=\{" " $\}$;

unsigned char $\operatorname{str} 2[]=\{" \quad "\}$;

sbit $\mathrm{TRH}=\mathrm{P}^{\wedge} 3$;

unint8 TH_data,TL_data,RH_data,RL_data,CK_data;

unint8 TH_temp,TL_temp, RH_temp,RL_temp,CK_temp;

unint8 com_data,untemp,temp;

unint 8 respond;

bit beep_st;

uchar $\mathrm{x}=0$;

int $\mathrm{m}$;

void main()

\{

InitTimer();

lcd_init();

DelayMs(15);

$\mathrm{BEEP}=0$;

$\mathrm{JDQ}=1$;

$\mathrm{DJ}=1$;

while(1)

$\{$ read_TRH();

checkkey();

if(set_st==0)

\{ 


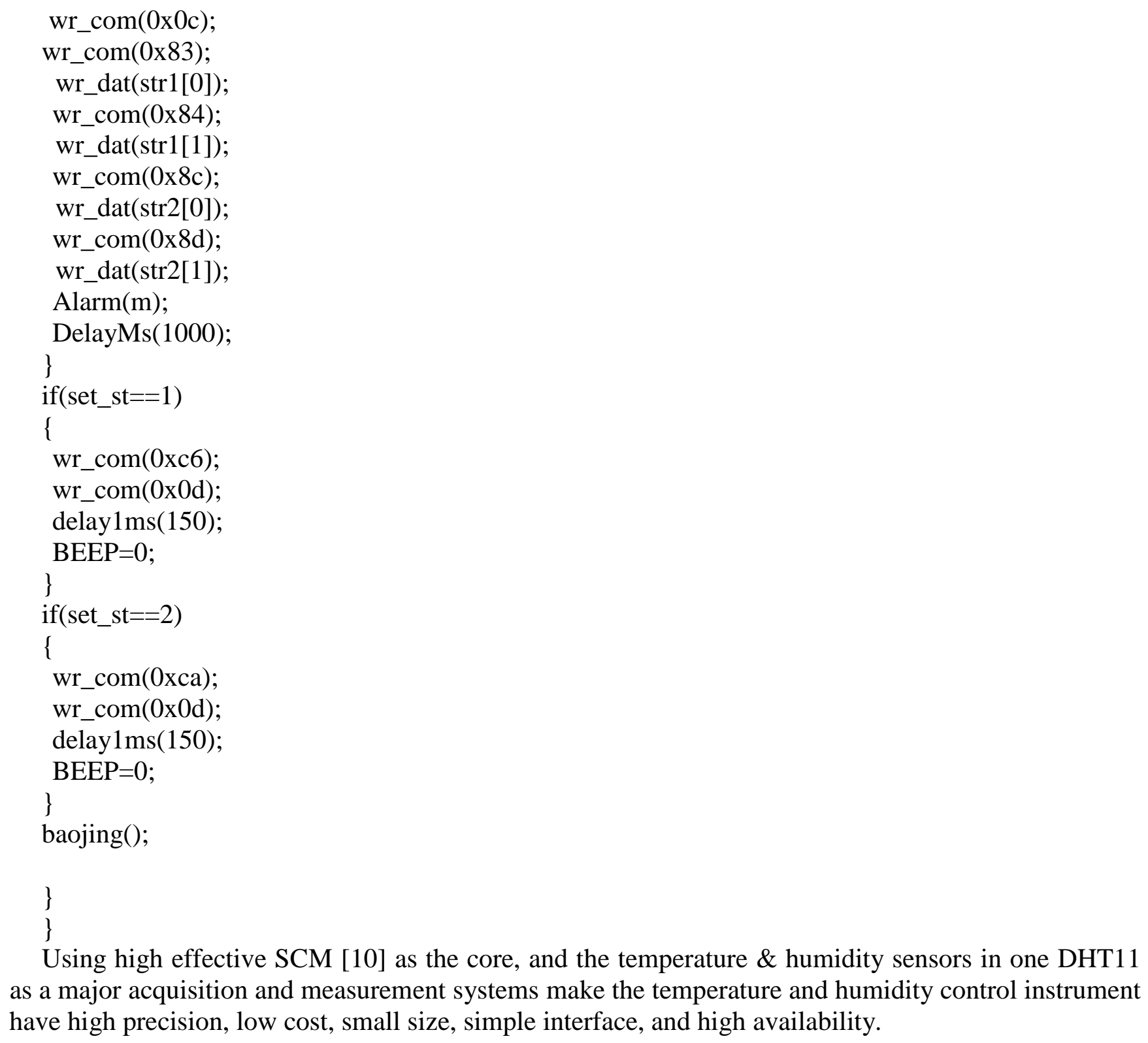

Using high effective SCM [10] as the core, and the temperature \& humidity sensors in one DHT11 as a major acquisition and measurement systems make the temperature and humidity control instrument have high precision, low cost, small size, simple interface, and high availability.

\section{References}

[1] He Xicai: Sensor and its application. [M] Beijing: National Defense Industry Press, 2011

[2] Zhang Yikun: Chip microcomputer principle and application [Z] Shan xi: Xi'an University of Electronic Science and Technology Press.

[3] Zhou Hangci: MCU application design techniques. [M] Beijing: Beijing University of Aeronautics and Astronautics Press, 2010.

[4] Yu Xicun: SCM principles and interface technology [Z] Shan xi: Xi'an University of Electronic Science and Technology Press .2012.12

[5] Yu Cchengbo: Sensor and automatic detection technology [Z] Beijing: Higher Education Press, 2009.7:108 to 112 
[6] Cao Hanfang: Digital circuits and logic design basis [Z] Beijing: Electronic Industry Press, 2007.7:119 to 120

[7] Tan Haoqiang: C Programming [Z] Beijing: Tsinghua University Press, 2005.7:95 121

[8] Zhang Yigang: MCS-51 Single-chip Application Design [M] Harbin: Harbin Institute of University Press .2013:142-169

[9] Jia Bonian, Yu Pu: Sensor Technology [M]. Southeast University Press, 2008:33-62

[10]Li Daohua, Li Ling, Zhu Yan: Sensor Circuit Analysis [M], Wuhan: Wuhan University Press, 2010:61-88 